\title{
Topological bifurcations in the evolution of coherent structures in a convection model
}

Dam, Magnus; Rasmussen, Jens Juul; Naulin, Volker; Brøns, Morten

Published in:

Physics of Plasmas

Link to article, DOI:

$10.1063 / 1.4993613$

Publication date:

2017

Document Version

Publisher's PDF, also known as Version of record

Link back to DTU Orbit

Citation (APA):

Dam, M., Rasmussen, J. J., Naulin, V., \& Brøns, M. (2017). Topological bifurcations in the evolution of coherent structures in a convection model. Physics of Plasmas, 24(8), [082301]. https://doi.org/10.1063/1.4993613

\section{General rights}

Copyright and moral rights for the publications made accessible in the public portal are retained by the authors and/or other copyright owners and it is a condition of accessing publications that users recognise and abide by the legal requirements associated with these rights.

- Users may download and print one copy of any publication from the public portal for the purpose of private study or research.

- You may not further distribute the material or use it for any profit-making activity or commercial gain

- You may freely distribute the URL identifying the publication in the public portal 
Topological bifurcations in the evolution of coherent structures in a convection model

Magnus Dam, Jens Juul Rasmussen, Volker Naulin, and Morten Brøns

Citation: Physics of Plasmas 24, 082301 (2017); doi: 10.1063/1.4993613

View online: http://dx.doi.org/10.1063/1.4993613

View Table of Contents: http://aip.scitation.org/toc/php/24/8

Published by the American Institute of Physics

\section{Articles you may be interested in}

Nonlinear parity mixtures controlling the propagation of interchange modes

Physics of Plasmas 24, 082501 (2017); 10.1063/1.4993472

Alfvén eigenmode stability and critical gradient energetic particle transport using the Trapped-Gyro-LandauFluid model

Physics of Plasmas 24, 072305 (2017); 10.1063/1.4989716

Impact of a hollow density profile on turbulent particle fluxes: Gyrokinetic and fluid simulations

Physics of Plasmas 24, 072303 (2017); 10.1063/1.4990078

Influence of plasma resistivity on peeling-ballooning modes

Physics of Plasmas 24, 062108 (2017); 10.1063/1.4985324

ExB mean flows in finite ion temperature plasmas

Physics of Plasmas 24, 062309 (2017); 10.1063/1.4985329

Beam-plasma instability and density holes: Langmuir wave-packet formation and particle acceleration Physics of Plasmas 24, 072103 (2017); 10.1063/1.4989724

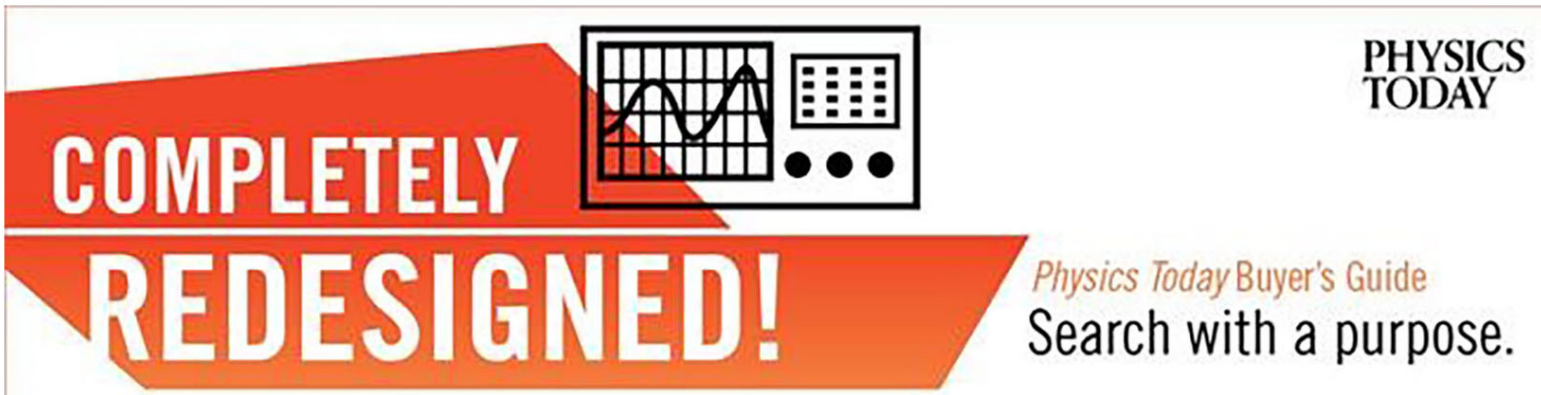




\title{
Topological bifurcations in the evolution of coherent structures in a convection model
}

\author{
Magnus Dam, ${ }^{1}$ Jens Juul Rasmussen, ${ }^{2}$ Volker Naulin, ${ }^{2}$ and Morten Brøns ${ }^{1}$ \\ ${ }^{1}$ Department of Applied Mathematics and Computer Science, Technical University of Denmark, \\ DK-2800 Kgs. Lyngby, Denmark \\ ${ }^{2}$ Department of Physics, Technical University of Denmark, DK-2800 Kgs. Lyngby, Denmark
}

(Received 1 May 2017; accepted 29 June 2017; published online 18 July 2017)

\begin{abstract}
Blob filaments are coherent structures in a turbulent plasma flow. Understanding the evolution of these structures is important to improve magnetic plasma confinement. Three state variables describe blob filaments in a plasma convection model. A dynamical systems approach analyzes the evolution of these three variables. A critical point of a variable defines a feature point for a region where that variable is significant. For a range of Rayleigh and Prandtl numbers, the bifurcations of the critical points of the three variables are investigated with time as the primary bifurcation parameter. Bifurcation curves separate the parameter planes into regions with different critical point configurations for the state variables. For Prandtl number equal to 1, the number of critical points of each state variable increases with increasing Rayleigh number. For Rayleigh number equal to $10^{4}$, the number of critical points is the greatest for Prandtl numbers of magnitude $10^{\circ}$. Published by AIP Publishing. [http://dx.doi.org/10.1063/1.4993613]
\end{abstract}

\section{INTRODUCTION}

The edge transport of a magnetically confined plasma is dominated by recurring bursts of coherent plasma structures. To improve the plasma confinement, it is crucial to understand the evolution of these structures. The plasma structures are in the low confinement (L-mode) regime known as blob filaments (blobs) and in the high confinement (H-mode) regime categorized as either edge localized mode (ELM) filaments or inter-ELM filaments. ${ }^{1}$ Blobs are localized meso-scale coherent structures in a turbulent flow, which are significantly denser and hotter than the surrounding plasma. They are highly localized in the plane perpendicular to the magnetic field and elongate along the magnetic field. ${ }^{2}$ Blobs form at the outboard mid-plane near the separatrix. The interchange instability causes the formation of finger-like structures that detach from the core plasma and create blobs. ${ }^{3-6}$ The grad- $B$ and curvature drifts, caused by the nonuniform magnetic field, charge polarize the blobs perpendicular to the directions of the magnetic field and the magnetic field variation. The resulting electric field generates an $\boldsymbol{E} \times \boldsymbol{B}$-drift causing the blob to propagate in the radially outward direction. The blobs propagate far into the scrape-off-layer (SOL) and increase unwanted plasma-wall interactions. During propagation in the SOL, the blobs deform and may lose coherence. ${ }^{7-20}$ The inclusion of finite Larmor radius effects in simulations enhances the blob coherence. $^{21-24}$

References 25-27 investigate the dependency of the blob position, velocity, and amplitude on Rayleigh and Prandtl numbers in a convection model. To describe the blob evolution, they apply the common practice of visual interpretation of time instant plots of the state variables. In fluid flows, the streamline topology and the vortices can be analyzed using dynamical systems theory. ${ }^{28-32}$ This method can analyze the topology of level curves of any function in the plane.
The present paper applies this dynamical systems approach to quantitatively describe the evolution of plasma blobs. A plasma convection model describes the evolution of seeded blobs. The electrostatic potential $\phi$, the thermodynamic variable $\theta$, and the vorticity $\Omega$ are the two-dimensional state variables describing the blobs. An extremum of a variable defines a feature point for a region where that variable is significant. ${ }^{33}$ Critical points of the electrostatic potential are instantaneous stagnation points for the unsteady plasma flow. Maxima of the thermodynamic variable are feature points for the blob, and the creation of additional maxima indicates that the blob splits into smaller blobs. Extrema of vorticity are feature points for vortices. For a range of Rayleigh and Prandtl numbers, we determine for each of the state variables $\phi, \theta$, and $\Omega$ the critical points and their type. We track the evolution, creation, and annihilation of the critical points as time increases.

\section{BIFURCATIONS OF STRUCTURES}

Let $H: M \longmapsto \mathbb{R}$ be any analytically or numerically given function defined on a subset of the plane $M \subseteq \mathbb{R}^{2}$. We assume that $H$ also depends on time $t$ and possibly some system parameters. For our application, $H$ represents the electrostatic potential $\phi$, the thermodynamic variable $\theta$, or the vorticity $\Omega$ given numerically from simulations. We fix all parameters and consider a single time instant. We consider $H(x, y)$ as a Hamiltonian for the autonomous Hamiltonian system

$$
\frac{d x}{d s}=\frac{\partial H}{\partial y}, \quad \frac{d y}{d s}=-\frac{\partial H}{\partial x} .
$$

The phase curves of the Hamiltonian system (1) lie on the level curves of $H$, 


$$
\frac{d H}{d s}=\frac{\partial H}{\partial x} \frac{d x}{d s}+\frac{\partial H}{\partial y} \frac{d y}{d s}=\frac{\partial H}{\partial x} \frac{\partial H}{\partial y}-\frac{\partial H}{\partial y} \frac{\partial H}{\partial x}=0 .
$$

A collection of level curves of $H$ comprises the Hamiltonian contour pattern. A point is an equilibrium of system (1), if and only if it is a critical point of $H$. A saddle of $H$ is a saddle point for the corresponding Hamiltonian system (1), while a local extremum of $H$ is a center for the corresponding Hamiltonian system (1).

We want to analyze the bifurcations of the critical points of $H$. We consider the time as the primary bifurcation parameter and the system parameters as secondary bifurcation parameters. In the analysis, we encounter three types of bifurcations. ${ }^{29,34,35}$ Figure 1(a) shows the saddle-center bifurcation, where a saddle and a center appear simultaneously through a cusp singularity as a bifurcation parameter $\mu$ is varied. This bifurcation is the Hamiltonian analogue to a saddle-node bifurcation. A Hamiltonian with the symmetry $H(x, y)=H(x,-y)$ allows the Hamiltonian pitchfork bifurcation in Fig. 1(b), where a center bifurcates into a saddle and two centers. A Hamiltonian with the symmetry $H(x, y)=-H(x,-y)$ allows the bifurcation in Fig. 1(c), where two saddles and two centers appear simultaneously through a degenerate saddle. We refer to this bifurcation as the duplex saddle-center bifurcation.

The topological property called the Poincaré index is a useful tool to keep track of equilibria during bifurcations. The index of a node, focus, or center is +1 and the index of a saddle is -1 . The sum of the indices of the equilibria remains constant during a bifurcation. ${ }^{36}$ To keep track of the bifurcations we follow all critical points even when only some types of critical points are of physical interest.

\section{CONVECTION MODEL}

We consider viscous plasma flow in a rectangular domain at the edge of a magnetically confined plasma in the
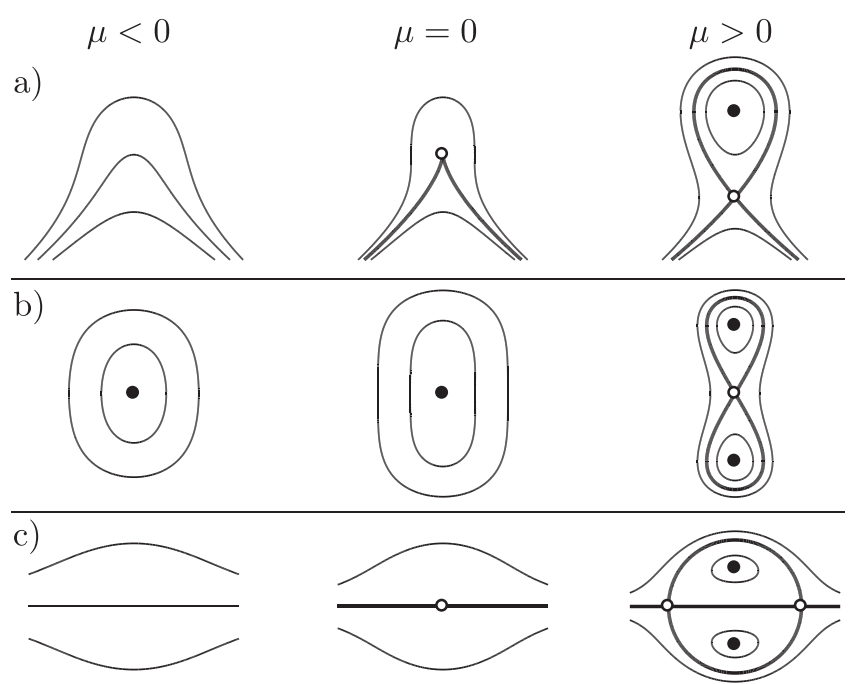

FIG. 1. The Hamiltonian contour patterns during (a) the saddle-center bifurcation, (b) the Hamiltonian pitchfork bifurcation, (c) the duplex saddlecenter bifurcation. The left panel $(\mu<0)$ is before the bifurcation, the middle panel $(\mu=0)$ is at the bifurcation, and the right panel $(\mu>0)$ is after the bifurcation. Filled dots are Lyapunov stable equilibria and unfilled dots are unstable equilibria. Thick lines are separatrices. plane perpendicular to the magnetic field $\boldsymbol{B}=B_{0} \boldsymbol{e}_{z}$. The flow is described using Cartesian coordinates $(x, y) \in M$, where $M=\left[-\frac{2}{5} L_{x}, \frac{3}{5} L_{x}\right] \times\left[-\frac{1}{2} L_{y}, \frac{1}{2} L_{y}\right]$. The normalized $\boldsymbol{E} \times \boldsymbol{B} \mathrm{drift}$ velocity field $\boldsymbol{v}=\left(v_{x}, v_{y}\right)^{\top}$ is

$$
\boldsymbol{v}=\left(\boldsymbol{e}_{z} \times \nabla \phi\right)_{\perp}=\left(\begin{array}{c}
-\partial_{y} \phi \\
\partial_{x} \phi
\end{array}\right) .
$$

Let $\Omega=\partial_{x} v_{y}-\partial_{y} v_{x}$ be the scalar vorticity. Then, the normalized electrostatic potential $\phi(x, y, t)$ is obtained from

$$
\nabla_{\perp}^{2} \phi=\Omega .
$$

To describe the evolution of a generic thermodynamic variable $\theta(x, y, t)$ (e.g., density, pressure, or temperature) and the vorticity $\Omega(x, y, t)$, we employ a normalized convection model

$$
\begin{gathered}
\left(\frac{\partial}{\partial t}+\boldsymbol{v} \cdot \nabla_{\perp}\right) \theta=\kappa \nabla_{\perp}^{2} \theta \\
\left(\frac{\partial}{\partial t}+\boldsymbol{v} \cdot \nabla_{\perp}\right) \Omega+\frac{\partial \theta}{\partial y}=\nu \nabla_{\perp}^{2} \Omega .
\end{gathered}
$$

Here, $\kappa$ is the nondimensional diffusion coefficient and $\nu$ is the nondimensional viscosity. These coefficients are related to Rayleigh and Prandtl numbers by $\mathrm{Ra}=1 /(\kappa \nu)$ and $\operatorname{Pr}$ $=\nu / \kappa$. We apply zero Dirichlet boundary conditions on all four boundaries for each of the variables $\phi, \theta$, and $\Omega$. The thermodynamic variable is initialized as a Gaussian function, $\theta(x, y, 0)=\exp \left(-\frac{1}{2}\left(x^{2}+y^{2}\right)\right)$, while the vorticity and the electrostatic potential are initialized to zero, $\phi(x, y, 0)$ $=\Omega(x, y, 0)=0$.

In Eq. (2), the thermodynamic variable has the reflection symmetry $\theta(x, y, t)=\theta(x,-y, t)$, while the electrostatic potential and the vorticity satisfy the symmetry $\phi(x, y, t)$ $=-\phi(x,-y, t)$ and $\Omega(x, y, t)=-\Omega(x,-y, t)$. These symmetries, together with the symmetric initial conditions, imply that $\theta$ is symmetric, while $\Omega$ and $\phi$ are antisymmetric for all times. System (2) is one of the simplest models used to describe nonlinear plasma dynamics. References 25-27 model the evolution of plasma blobs with system (2), while Refs. 7-10 also use similar convection models also to describe plasma blob evolution.

\section{NUMERICAL METHOD}

We define the size of the computational domain by $L_{x}=L_{y}=50$. This is sufficiently large to ensure that the evolution of the blobs is insignificantly affected by the boundary conditions. To obtain the simulation data, we first fix $\operatorname{Pr}=1$ and solve the convection problem (2) for numerous Rayleigh numbers. Then, we fix $\mathrm{Ra}=10^{4}$ and solve problem (2) for numerous Prandtl numbers.

The FEM software package COMSOL Multiphysics ${ }^{\circledR}$ is used as the numerical solver. ${ }^{37}$ To obtain the required simulation results with sufficient precision, we use a triangular mesh and activate the adaptive mesh refinement, which automatically refines the mesh in regions with large gradients of $\theta$ or $\Omega$. The maximum number of mesh refinement iterations 
is set to 10 . This gives a mesh containing approximately $3 \times 10^{5}$ domain elements. The convection problem is initialized at $t=0$ and runs with output time steps of $\Delta t=0.05$ until $t=20$. The mesh reinitializes and iteratively refines, at $t=0,2, \ldots, 18$, for a total of 10 times during each simulation.

In the analysis, we track the evolution, creation, and annihilation of the critical points of $\phi, \theta$, and $\Omega$. The eigenvalues determine whether a critical point is a saddle, maximum, or minimum. For each simulation, we determine the critical points of $\phi, \theta$, and $\Omega$ numerically as follows: The nullclines of the Hamiltonian system (1) are

$$
\begin{aligned}
& N_{x}=\left\{(x, y) \in M \mid \partial_{y} H(x, y)=0\right\}, \\
& N_{y}=\left\{(x, y) \in M \mid \partial_{x} H(x, y)=0\right\} .
\end{aligned}
$$

Here, $H$ represents either $\phi, \theta$, or $\Omega$ at a fixed time $t$. The set of critical points of $H$ is $N_{x} \cap N_{y}$. To numerically determine the set of critical points, we first compute $N_{y}$. Points in regions where $\theta$ is smaller than $1 \%$ of $\theta_{\max }$ $=\max _{(x, y) \in M} \theta(x, y, t)$ are removed from $N_{y}$. These regions contain very few particles or little energy and are therefore physically uninteresting. The nullcline set $N_{y}$ consists of a number of parametrized curve segments $\gamma_{i}(s), i=1, \ldots, N$. Along each curve segment we compute $\partial_{y} H\left(\gamma_{i}(s)\right)$ and determine the values of $s_{j}$ for which $\partial_{y} H\left(\gamma_{i}\left(s_{j}\right)\right)=0$. The corresponding points $\gamma_{i}\left(s_{j}\right)$ are the critical points of $H$. The type of a critical point of $H$ is determined from the eigenvalues $\lambda_{1,2}$ of the Hessian matrix of $H$ evaluated in that point

$$
\mathcal{D}^{2} H=\left(\begin{array}{ll}
H_{x x} & H_{x y} \\
H_{y x} & H_{y y}
\end{array}\right) .
$$

Since $\mathcal{D}^{2} H$ is symmetric, both eigenvalues are real. The critical point is a saddle if $\lambda_{1}$ and $\lambda_{2}$ are of opposite sign, a local maximum if $\lambda_{1,2}$ are both negative, a local minimum if $\lambda_{1,2}$ are both positive, and a degenerate point if either $\lambda_{1}$ or $\lambda_{2}$ is zero.

\section{BIFURCATION ANALYSIS}

This section analyzes the bifurcations of the critical points of the electrostatic potential $\phi$, the thermodynamic variable $\theta$, and the vorticity $\Omega$ as time increases from $t=0$ to $t=20$ for a range of Rayleigh and Prandtl numbers. We use the same set of simulation data for the analysis of $\phi, \theta$, and $\Omega$. We first fix $(\mathrm{Pr}, \mathrm{Ra})=\left(1,10^{4}\right)$ and track the evolution, creation, and annihilation of the critical points as time evolves. We then fix $\operatorname{Pr}=1$ and track the creation and annihilation of the critical points for numerous Rayleigh numbers in the range $\mathrm{Ra} \in\left[10,10^{5}\right]$. Similarly, we fix $\mathrm{Ra}=10^{4}$ and track the creation and annihilation of the critical points for numerous Prandtl numbers in the range $\operatorname{Pr} \in\left[10^{-3}, 10^{4}\right]$. We draw the bifurcation curves in the $(t, \mathrm{Ra})$ - and $(t, \mathrm{Pr})$-parameter planes.

\section{A. Critical points of the electrostatic potential}

The electrostatic potential defines the velocity field of a plasma flow analogous to the way the stream function

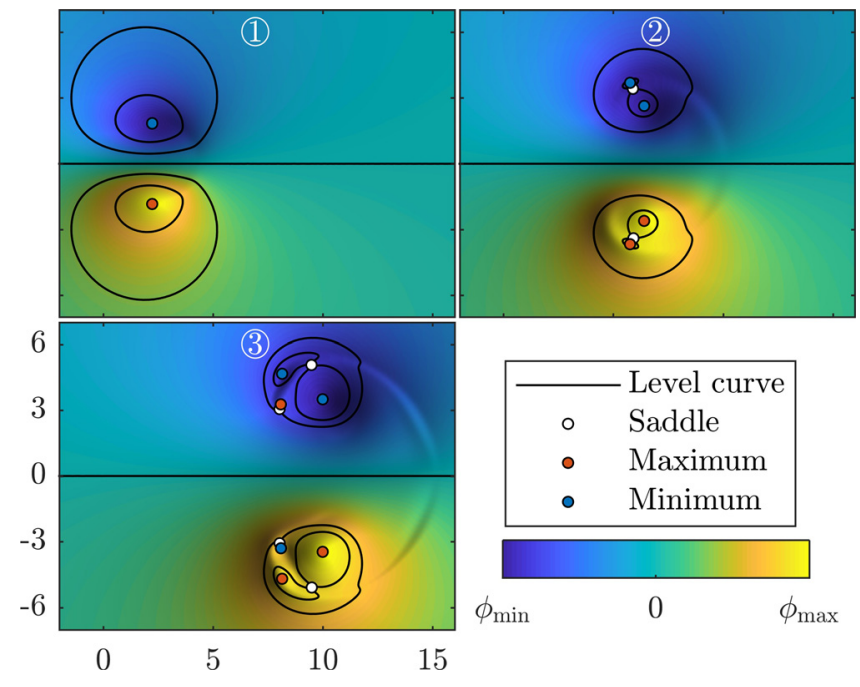

FIG. 2. Representative critical point configurations for $\phi$ corresponding to different level curve topologies of $\phi$.

defines the velocity field of a fluid flow. The critical points of $\phi$ define the instantaneous stagnation points of the flow.

Figure 2 shows representative time instant plots of $\phi$ for three different critical point configurations labeled (1)-(3) superimposed by the contour patterns of $\phi$. The symmetry $\phi(x, y, t)=-\phi(x,-y, t)$ causes the $x$-axis to be the zero level set and imposes a symmetry of the critical points such that we can limit our comments to bifurcations in the upper half-plane (uhp). Table I characterizes the different critical point configurations of $\phi$ by the number of each type of critical point.

For $(\operatorname{Ra}, \operatorname{Pr})=\left(10^{4}, 1\right)$, we consider $\phi(x, y, t)$ with time $t$ $\in[0,20]$ as a bifurcation parameter. The bifurcation diagram in Fig. 3 shows the $y$-coordinates of the critical points of $\phi$ as a function of $t$. The electrostatic potential $\phi$ is initialized to zero, but quickly a minimum emerges at $(x, y) \approx(0,1.58)$ such that $\phi$ has the configuration (1). At $t=10.13$, a saddlecenter bifurcation creates a saddle and a minimum changing the configuration to (2). At $t=14.91$, this saddle and minimum vanish again in a saddle-center bifurcation and $\phi$ again has the configuration (1).

We fix $\operatorname{Pr}=1$, and for numerous Rayleigh numbers, we determine all bifurcation values of $t$ for the critical points of $\phi$. Then, we fix $\mathrm{Ra}=10^{4}$, and for numerous Prandtl numbers, we determine all the bifurcation values of $t$. Figure 4 shows the bifurcation curves in the $(t, \mathrm{Ra})$ - and $(t, \mathrm{Pr})$-planes. Two types of saddle-center bifurcations are observed: The saddle-center bifurcation ( $\mathrm{min}$ ) creates or annihilates a saddle and a minimum in the upper half-plane, while the saddle-

TABLE I. Number of saddles, maxima (max), and minima (min) in the upper half-plane (uhp) and the lower half-plane (lhp) for different critical point configurations of $\phi$ shown in Fig. 2. The index +2 is preserved.

\begin{tabular}{llll}
\hline \hline Critical points of $\phi$ & (1) & (2) & (3) \\
\hline Saddles in uhp and lhp & 0 & 1 & 2 \\
Max in uhp and min in lhp & 0 & 0 & 1 \\
Min in uhp and max in lhp & 1 & 2 & 2 \\
Total (uhp + lhp) & 2 & 6 & 10 \\
\hline \hline
\end{tabular}




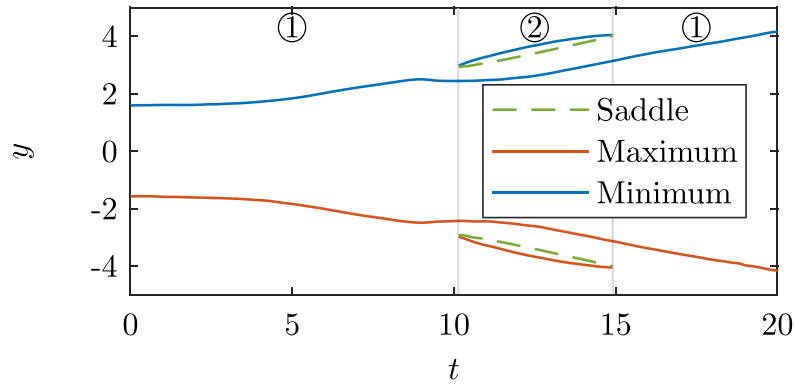

FIG. 3. Bifurcation diagram showing the $y$-coordinates of all critical points of $\phi$ for $(\mathrm{Ra}, \mathrm{Pr})=\left(10^{4}, 1\right)$ and time as a bifurcation parameter. Saddlecenter bifurcations occur at $t=10.13$ and $t=14.91$. The different critical point configurations of $\phi$ are shown in Fig. 2.

center bifurcation $(\max )$ creates or annihilates a saddle and a maximum in the upper half-plane. The bifurcation curves divide these sections of the parameter planes into regions with configurations (1)-3). For fixed $\operatorname{Pr}=1$, no bifurcations occur for Ra $\leqslant 5.1 \times 10^{3}$. The number of critical points of $\phi$ increases with increasing Rayleigh number. For fixed $\mathrm{Ra}=10^{4}$, no bifurcations occur for $\operatorname{Pr} \leqslant 3.0 \times 10^{-1}$ or $\operatorname{Pr} \gtrsim 9.0$.

\section{B. Critical points of the thermodynamic variable}

The thermodynamic variable defines the physical shape of the blob. A maximum of $\theta$ is a feature point for the blob. The creation of additional maxima indicates that the blob splits into smaller blobs. We can use the number of maxima of $\theta$ as a measure for the level of coherence of a blob. A blob with a single maximum is a fully coherent blob and a blob with more maxima is less coherent.

Figure 5 shows representative time instant plots of $\theta$ for different critical point configurations labeled (1)-8. Table II characterizes the different configurations of $\theta$ by the number of each type of critical point.

The bifurcation diagram in Fig. 6 shows for (Ra, $\operatorname{Pr})=\left(10^{4}, 1\right)$ the $y$-coordinates of the critical points of $\theta$ as a function of $t$. The thermodynamic variable $\theta$ is initialized with a maximum at $(x, y)=(0,0)$ corresponding to the

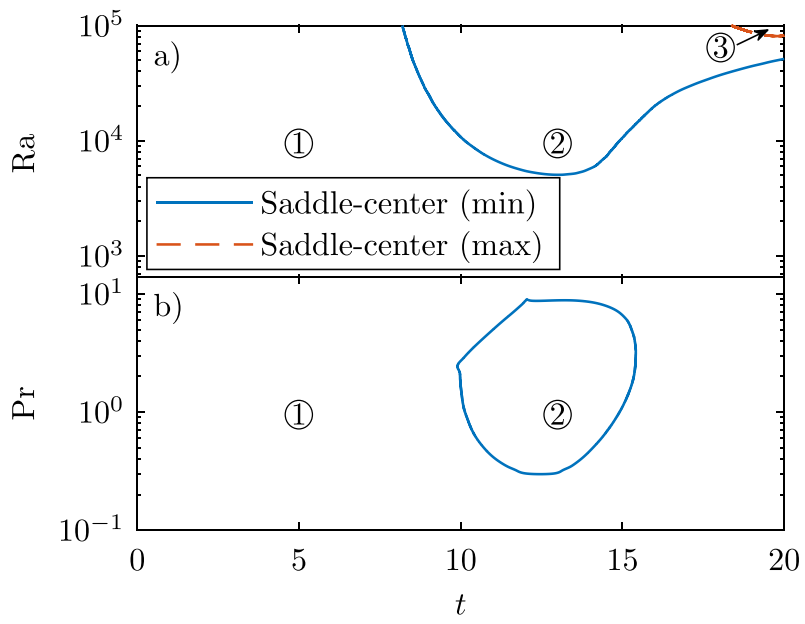

FIG. 4. Bifurcation curves for the critical points of $\phi$ in a) the $(t, \mathrm{Ra})$-parameter plane for $\operatorname{Pr}=1, \mathrm{~b})$ the $(t, \mathrm{Pr})$-parameter plane for $\mathrm{Ra}=10^{4}$. The different critical point configurations of $\phi$ are shown in Fig. 2.
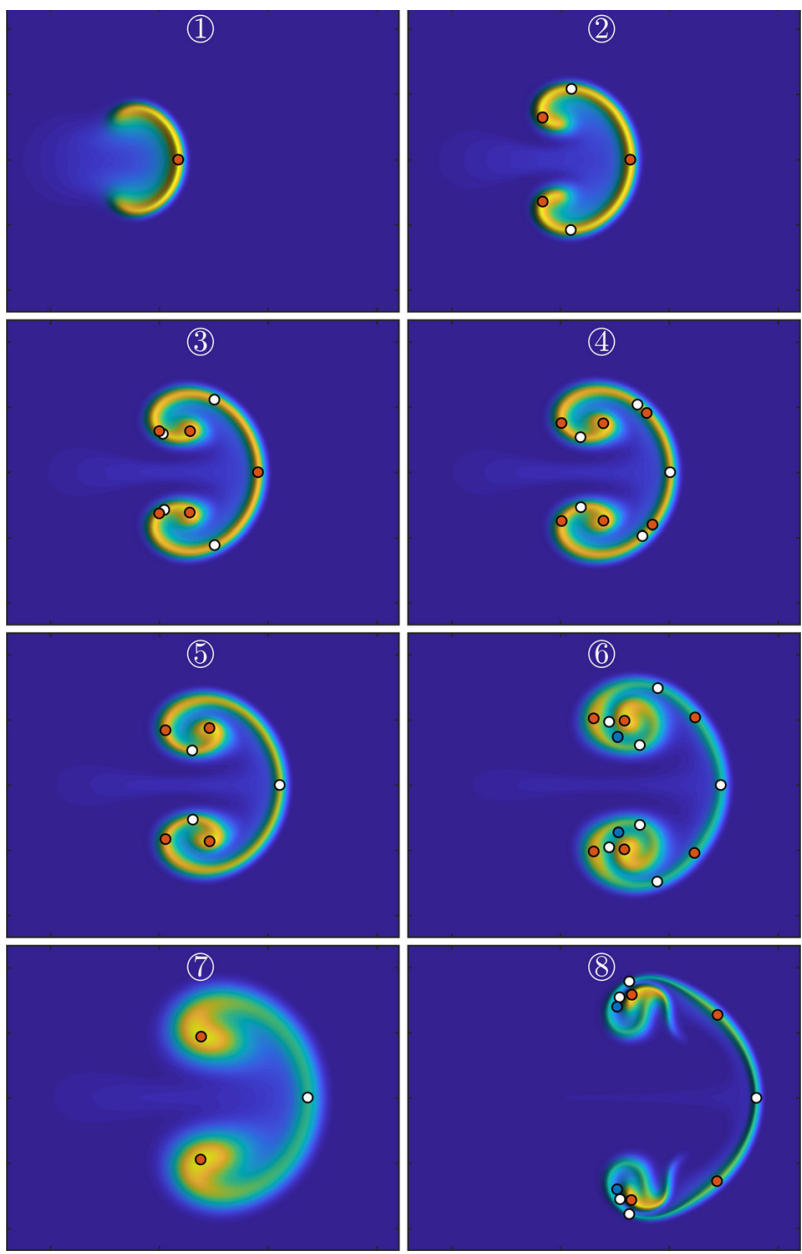

FIG. 5. Representative critical point configurations for $\theta$.

critical point configuration (1). Saddle-center bifurcations at $t=8.55$ and $t=11.69$ change the critical point configuration to (2) and further to (3). At $t=11.91$, a Hamiltonian pitchfork bifurcation, allowed by the reflection symmetry, changes the critical point configuration to (4). Four more saddle-center bifurcations bring the critical point configuration through (5)-(4)-(6)-(4)

Figure 7 shows the bifurcation curves in the (t, Ra)- and $(t, \mathrm{Pr})$-planes. We observe three types of bifurcations: The saddle-center bifurcation (max) creates or annihilates a saddle and a maximum in the upper and lower half-planes (lhp), the saddle-center bifurcation $(\mathrm{min})$ creates or annihilates a saddle and a minimum in the upper and lower half-planes, and the Hamiltonian pitchfork bifurcation splits a maximum

TABLE II. Number of saddles, maxima (max), and minima (min) in the upper half-plane (uhp) and the lower half-plane (lhp) for different critical point configurations of $\theta$ shown in Fig. 5. The index +1 is preserved.

\begin{tabular}{lllllllll}
\hline \hline Critical points of $\theta$ & (1) & (2) & (3) & (4) & (5) & (6) & (7) & (8) \\
\hline Saddles in uhp and lhp & 0 & 1 & 2 & 2 & 1 & 3 & 0 & 2 \\
Max in uhp and lhp & 0 & 1 & 2 & 3 & 2 & 3 & 1 & 2 \\
Min in uhp and lhp & 0 & 0 & 0 & 0 & 0 & 1 & 0 & 1 \\
Saddles on the $x$-axis & 0 & 0 & 0 & 1 & 1 & 1 & 1 & 1 \\
Max on the $x$-axis & 1 & 1 & 1 & 0 & 0 & 0 & 0 & 0 \\
Total (uhp + lhp $+x$-axis) & 1 & 5 & 9 & 11 & 7 & 15 & 3 & 11 \\
\hline
\end{tabular}




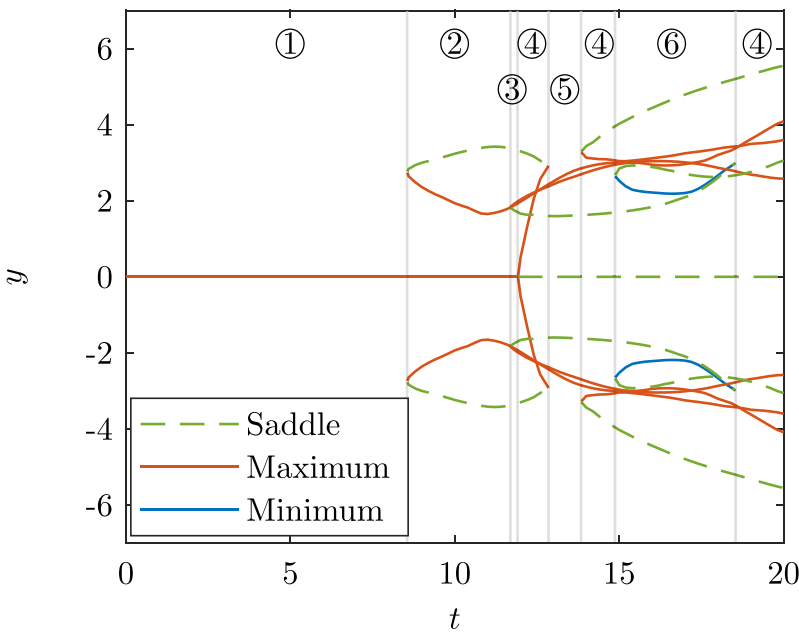

FIG. 6. Bifurcation diagram showing the $y$-coordinates of all critical points of $\theta$ for $(\mathrm{Ra}, \operatorname{Pr})=\left(10^{4}, 1\right)$ and time as a bifurcation parameter. Saddlecenter bifurcations occur at $t=8.55,11.69,12.85,13.84,14.87$, and 18.54, and a Hamiltonian pitchfork bifurcation occurs at $t=11.91$. The different critical point configurations of $\theta$ are shown in Fig. 5.

on the $x$-axis into a saddle on the $x$-axis and two maxima symmetrically located in the upper and lower half-planes. The first bifurcation marks the time the blob starts to lose coherence. For $\operatorname{Pr}=1$, no bifurcations occur for $\mathrm{Ra} \leq 5.3 \times 10^{1}$. The number of critical points increases with increasing Rayleigh number. For $\mathrm{Ra}=10^{4}$, no bifurcations occur for $\operatorname{Pr} \lesssim 1.1 \times 10^{-3}$ or $\operatorname{Pr} \gtrsim 1.5 \times 10^{3}$. The number of critical points of $\theta$ is the greatest for Prandtl numbers of

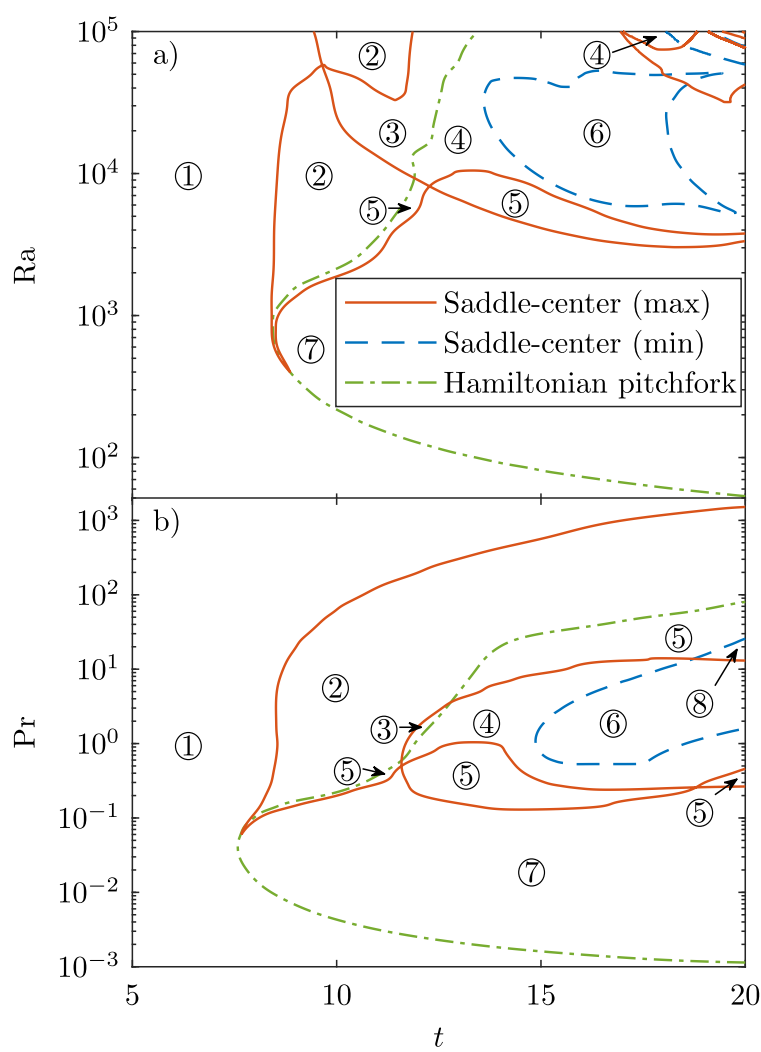

FIG. 7. Bifurcation curves for the critical points of $\theta$ in a) the $(t, \mathrm{Ra})$-parameter plane for $\operatorname{Pr}=1, \mathrm{~b})$ the $(t, \mathrm{Pr})$-parameter plane for $\mathrm{Ra}=10^{4}$. The different critical point configurations of $\theta$ are shown in Fig. 5. magnitude $10^{\circ}$. Hence, blobs stay more coherent for small Rayleigh numbers, and for small or large Prandtl numbers.

\section{Critical points of the vorticity}

The extrema of $\Omega$ define vortex centers and are feature points of the vortices. A typical vortex analysis follows the full evolution of the vortical regions enclosed by separatrices. Here, we simply track the evolution of the extrema of vorticity, and in this way, disregard all information about the actual vortex shape. The sign of vorticity in an extremum defines the direction of rotation of the vortex.

Figure 8 shows representative time instant plots of $\Omega$ for different critical point configurations labeled (1)_8. The symmetry $\Omega(x, y, t)=-\Omega(x,-y, t)$ causes the $x$-axis to be a zero level set and imposes a symmetry of the critical points such that we can limit our comments to bifurcations in the upper half-plane and on the $x$-axis. Table III characterizes the different configurations of $\Omega$ by the number of each type of critical point.

The bifurcation diagram in Fig. 9 shows for (Ra, $\operatorname{Pr})=\left(10^{4}, 1\right)$ the $y$-coordinates of the critical points of $\Omega$ as a function of $t$. The vorticity $\Omega$ is initialized to zero, but a maximum at $(x, y) \approx(0.0,1.0)$ quickly emerges giving $\Omega$ the critical point configuration (1). A duplex saddle-center bifurcation, allowed by the symmetry $\Omega(x, y, t)=-\Omega(x,-y, t)$, changes the configuration to (2). A series of saddle-center
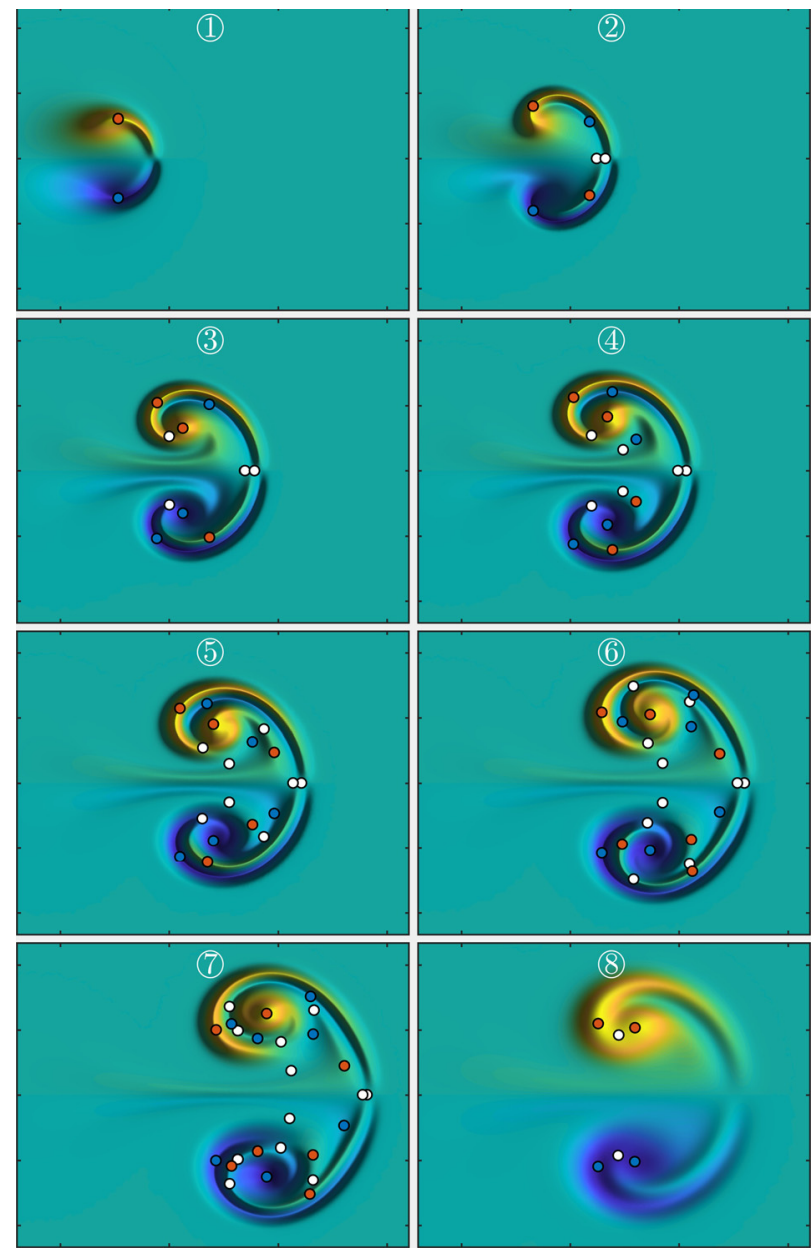

FIG. 8. Representative critical point configurations for $\Omega$. 
TABLE III. Number of saddles, maxima (max), and minima ( $\min$ ) in the upper half-plane (uhp) and the lower half-plane (lhp) for different critical point configurations of $\Omega$ shown in Fig. 8. The index +2 is preserved.

\begin{tabular}{lcccccccc}
\hline \hline Critical points of $\Omega$ & (1) & (2) & (3) & (4) & (5) & (6) & (7) & (8) \\
\hline Saddles in uhp and lhp & 0 & 0 & 1 & 2 & 3 & 4 & 5 & 1 \\
Max in uhp and min in lhp & 1 & 1 & 2 & 2 & 3 & 3 & 3 & 2 \\
Min in uhp and max in lhp & 0 & 1 & 1 & 2 & 2 & 3 & 4 & 0 \\
Saddles on the $x$-axis & 0 & 2 & 2 & 2 & 2 & 2 & 2 & 0 \\
Total (hhp + lhp $+x$-axis) & 2 & 6 & 10 & 14 & 18 & 22 & 26 & 6 \\
\hline
\end{tabular}

bifurcations then creates or annihilates saddles and extrema, which changes the critical point configuration through (3)-(4)-(5)-(6)-(7)-(6).

Figure 10 shows the bifurcation curves for the critical points of $\Omega$ in the ( $t, \mathrm{Ra})$ - and ( $t, \mathrm{Pr}$ )-planes. Three types of bifurcations are observed: The saddle-center bifurcation (max) creates or annihilates a saddle and a maximum in the upper half-plane, the saddle-center bifurcation (min) creates or annihilates a saddle and a minimum in the upper halfplane, and the duplex saddle-center (min) creates two saddles on the $x$-axis, a minimum in the upper half-plane and a symmetrically located maximum in the lower half-plane. For $\operatorname{Pr}=1$, no bifurcations occur for $\operatorname{Ra} \leqslant 6.5 \times 10^{2}$. For $\mathrm{Ra}=10^{4}$, no bifurcations occur for $\operatorname{Pr} \leqslant 8.0 \times 10^{-3}$ or $\mathrm{Ra} \gtrsim 2.8 \times 10^{3}$.

\section{Discussion}

The diagrams in Figs. 4, 7, and 10 qualitatively follow the same pattern: For $\operatorname{Pr}=1$, the number of critical points increases with increasing Rayleigh number and for $\mathrm{Ra}=10^{4}$, the number of critical points is the greatest for Prandtl numbers of magnitude $10^{\circ}$. The electrostatic potential $\phi$ at each time instant is related to the vorticity $\Omega$ by Eq. (2b). However, the vorticity has many more critical points than the electrostatic potential. A quantitative comparison of the three diagrams show no direct relation between the

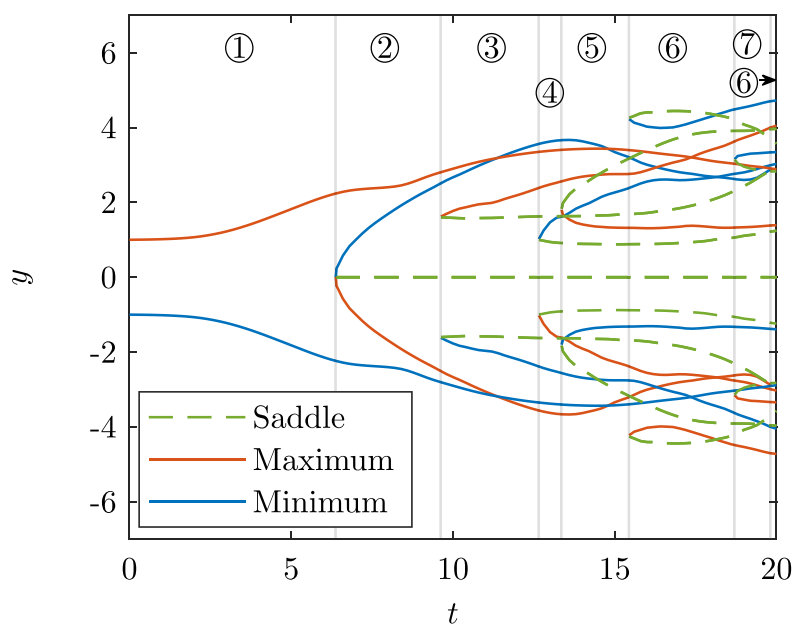

FIG. 9. Bifurcation diagram showing the $y$-coordinates of all critical points of $\Omega$ for $(\mathrm{Ra}, \mathrm{Pr})=\left(10^{4}, 1\right)$ and time as a bifurcation parameter. A duplex saddle-center bifurcation occurs at $t=6.37$ and saddle-center bifurcations occur at $9.62,12.65,13.35,15.44,18.70$, and 19.81 . The different critical point configurations of $\Omega$ are shown in Fig. 8 .

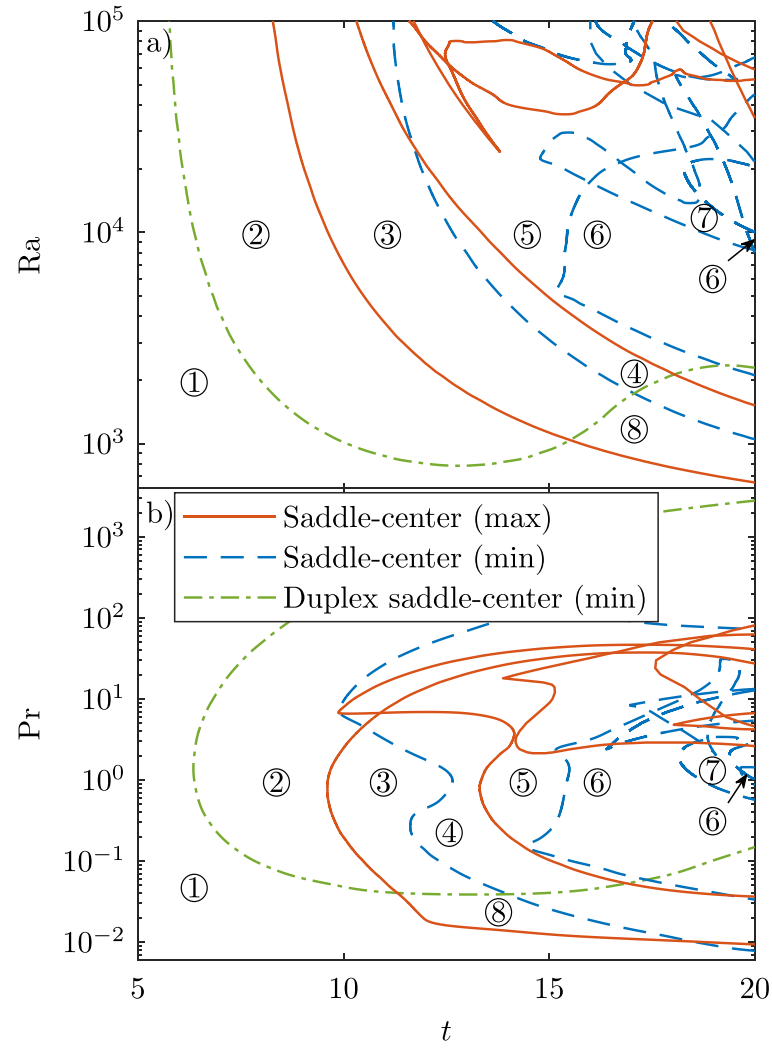

FIG. 10. Bifurcation curves for the critical points of $\Omega$ in a) the $(t, \mathrm{Ra})$ parameter plane for $\operatorname{Pr}=1, \mathrm{~b})$ the $(t, \operatorname{Pr})$-parameter plane for $\mathrm{Ra}=10^{4}$. The different critical point configurations of $\Omega$ are shown in Fig. 8 .

bifurcation values of $t$ at which critical points are created or annihilated. Hence, the analysis of one state variable only characterizes the configurations of the feature points corresponding to that variable. The analysis restricts to $\mathrm{Ra} \leq 10^{5}$ and $t \leq 20$. By simultaneously increasing $\mathrm{Ra}$ and $t$ further beyond these values, the number of bifurcations grows to the extent that it becomes both impractical and of limited interest to systematically track and distinguish the different bifurcations. The present method is unsuitable to describe structures in such a turbulent flow.

\section{CONCLUSION}

A convection model, with Rayleigh number, $\mathrm{Ra}$, and Prandtl number, Pr, as parameters, describes the evolution of two-dimensional seeded plasma blobs. The blobs are described in terms of three variables: the electrostatic potential $\phi$, the thermodynamic variable $\theta$, and the vorticity $\Omega$. The critical points of a variable define feature points where that variable is significant. Extrema of the electrostatic potential define stagnation points. Maxima of the thermodynamic variable are feature points for the blob such that the creation of additional maxima indicates a splitting of the blob into smaller blobs. Extrema of vorticity are feature points for vortices.

We apply a dynamical systems approach to analyze bifurcations of the critical points of $\phi, \theta$, and $\Omega$ with time, $t$ $\in[0,20]$ as the primary bifurcation parameter. We fix the Prandtl number to $\operatorname{Pr}=1$ and consider the Rayleigh number as an additional bifurcation parameter. We then fix the 
Rayleigh number to $\mathrm{Ra}=10^{4}$ and use the Prandtl number as an additional bifurcation parameter. The bifurcation curves separate the parameter planes into multiple regions with different critical point configurations. The diagrams reveal that for $\operatorname{Pr}=1$, the number of critical points of $\phi, \theta$, and $\Omega$ increases for an increasing Rayleigh number. For $\mathrm{Ra}=10^{4}$, the number of critical points is the greatest for Prandtl numbers of magnitude $10^{\circ}$.

We have demonstrated that a bifurcation analysis of the critical points is a feasible method to quantitatively describe the evolution of coherent structures in a plasma physics convection model.

${ }^{1}$ W. Fundamenski, J. Nucl. Mater. 390-391, 10-19 (2009).

${ }^{2}$ D. A. D'Ippolito, J. R. Myra, and S. J. Zweben, Phys. Plasmas 18, 060501 (2011).

${ }^{3}$ O. E. Garcia, V. Naulin, A. H. Nielsen, and J. Juul Rasmussen, Phys. Scr. T122, 89-103 (2006).

${ }^{4} J$. R. Myra, W. M. Davis, D. A. D'Ippolito, B. LaBombard, D. A. Russell, J. L. Terry, and S. J. Zweben, Nucl. Fusion 53, 073013 (2013).

${ }^{5}$ P. Zhu, C. R. Sovinec, and C. C. Hegna, Phys. Plasmas 22, 022311 (2015).

${ }^{6}$ A. H. Nielsen, J. Juul Rasmussen, J. Madsen, G. S. Xu, V. Naulin, J. M. B. Olsen, M. Løiten, S. K. Hansen, N. Yan, L. Tophøj, and B. N. Wan, Plasma Phys. Controlled Fusion 59, 025012 (2017).

${ }^{7}$ A. Y. Aydemir, Phys. Plasmas 12, 062503 (2005).

${ }^{8}$ R. Kube and O. E. Garcia, Phys. Plasmas 18, 102314 (2011)

${ }^{9}$ R. Kube and O. E. Garcia, Phys. Plasmas 19, 042305 (2012).

${ }^{10}$ R. Kube, O. E. Garcia, and M. Wiesenberger, Phys. Plasmas 23, 122302 (2016).

${ }^{11}$ O. E. Garcia, N. H. Bian, V. Naulin, A. H. Nielsen, and J. Juul Rasmussen, Phys. Scr. T122, 104-124 (2006).

${ }^{12}$ J. R. Angus, S. I. Krasheninnikov, and M. V. Umansky, Phys. Plasmas 19, 082312 (2012).

${ }^{13}$ N. R. Walkden, B. D. Dudson, and G. Fishpool, Plasma Phys. Controlled Fusion 55, 105005 (2013).

${ }^{14}$ J. R. Angus and M. V. Umansky, Phys. Plasmas 21, 012514 (2014).

${ }^{15}$ F. D. Halpern, A. Cardellini, P. Ricci, S. Jolliet, J. Loizu, and A. Mosetto, Phys. Plasmas 21, 022305 (2014).

${ }^{16}$ L. Easy, F. Militello, J. Omotani, B. Dudson, E. Havlíčková, P. Tamain, V. Naulin, and A. H. Nielsen, Phys. Plasmas 21, 122515 (2014).
${ }^{17}$ W. Lee, M. V. Umansky, J. R. Angus, and S. I. Krasheninnikov, Phys. Plasmas 22, 012505 (2015).

${ }^{18}$ N. R. Walkden, B. D. Dudson, L. Easy, G. Fishpool, and J. T. Omotani, Nucl. Fusion 55, 113022 (2015).

${ }^{19}$ J. T. Omotani, F. Militello, L. Easy, and N. R. Walkden, Plasma Phys. Controlled Fusion 58, 014030 (2016).

${ }^{20}$ N. R. Walkden, L. Easy, F. Militello, and J. T. Omotani, Plasma Phys. Controlled Fusion 58, 115010 (2016).

${ }^{21}$ J. Madsen, O. E. Garcia, J. S. Larsen, V. Naulin, A. H. Nielsen, and J. Juul Rasmussen, Phys. Plasmas 18, 112504 (2011).

${ }^{22}$ M. Wiesenberger, J. Madsen, and A. Kendl, Phys. Plasmas 21, 092301 (2014).

${ }^{23}$ J. Olsen, J. Madsen, A. H. Nielsen, J. Juul Rasmussen, and V. Naulin, Plasma Phys. Controlled Fusion 58, 044011 (2016).

${ }^{24}$ M. Held, M. Wiesenberger, J. Madsen, and A. Kendl, Nucl. Fusion 56, 126005 (2016)

${ }^{25}$ N. Bian, S. Benkadda, J.-V. Paulsen, and O. E. Garcia, Phys. Plasmas 10, 671 (2003).

${ }^{26}$ O. E. Garcia, N. H. Bian, V. Naulin, A. H. Nielsen, and J. Juul Rasmussen, Phys. Plasmas 12, 090701 (2005).

${ }^{27}$ O. E. Garcia, N. H. Bian, and W. Fundamenski, Phys. Plasmas 13, 082309 (2006).

${ }^{28}$ P. G. Bakker, Bifurcations in Flow Patterns, Nonlinear Topics in the Mathematical Sciences (Springer, The Netherlands, 1991), Vol. 2.

${ }^{29}$ M. Brøns, L. K. Voigt, and J. N. Sørensen, J. Fluid Mech. 401, 275-292 (1999).

${ }^{30}$ M. Brøns, Adv. Appl. Mech. 41, 1-42 (2007).

${ }^{31}$ M. Brøns, B. Jacobsen, K. Niss, A. V. Bisgaard, and L. K. Voigt, J. Fluid Mech. 584, 23-43 (2007).

${ }^{32}$ M. Heil, J. Rosso, A. L. Hazel, and M. Brøns, J. Fluid Mech. 812, 199-221 (2017).

${ }^{33}$ J. Kasten, J. Reininghaus, I. Hotz, H. C. Hege, B. R. Noack, G. Daviller, and M. Morzyński, Arch. Mech. 68, 55-80 (2016).

${ }^{34} \mathrm{H}$. Broer, I. Hoveijn, G. Lunter, and G. Vegter, Bifurcations in Hamiltonian Systems, Lecture Notes in Mathematics (Springer, 2003), Vol. 1806.

${ }^{35}$ H. Hanßmann, Local and Semi-Local Bifurcations in Hamiltonian Dynamical Systems, Lecture Notes in Mathematics (Springer, 2007), Vol. 1893

${ }^{36}$ J. D. Meiss, Differential Dynamical Systems, Mathematical Modeling and Computation (Society for Industrial and Applied Mathematics, 2007), Vol. 14.

37“"COMSOL Multiphysics® v. 5.3," COMSOL AB, Stockholm, Sweden. 